\title{
Coronary artery height differences and their effect on fractional flow reserve
}

\author{
Firas Al-Janabi ${ }^{1,2}$, Grigoris Karamasis ${ }^{1,2}$, Christopher M. Cook ${ }^{3}$, Alamgir M. Kabir ${ }^{1}$, \\ Rohan O. Jagathesan ${ }^{1}$, Nicholas M. Robinson ${ }^{1}$, Jeremy W. Sayer ${ }^{1}$, \\ Rajesh K. Aggarwal ${ }^{1}$, Gerald J. Clesham ${ }^{1,2}$, Paul R. Kelly ${ }^{1}$, Reto A. Gamma ${ }^{1}$, \\ Kare H. Tang ${ }^{1}$, Thomas R. Keeble ${ }^{1,2}$, John R. Davies ${ }^{1,2}$
}

${ }^{1}$ Essex Cardiothoracic Center, Nethermayne, Basildon, Essex, United Kingdom ${ }^{2}$ Anglia Ruskin University, Bishop Hall Lane, Chelmsford, Essex, United Kingdom

${ }^{3}$ National Heart and Lung Institute, Dovehouse Street, London, United Kingdom

\begin{abstract}
Background: Fractional flow reserve (FFR) uses pressure-based measurements to assess the severity of a coronary stenosis. Distal pressure ( $P d)$ is often at a different vertical height to that of the proximal aortic pressure (Pa). The difference in pressure between Pd and Pa due to hydrostatic pressure, may impact FFR calculation.

Methods: One hundred computed tomography coronary angiographies were used to measure height differences between the coronary ostia and points in the coronary tree. Mean heights were used to calculate the hydrostatic pressure effect in each artery, using a correction factor of $0.8 \mathrm{mmHg} / \mathrm{cm}$. This was tested in a simulation of intermediate coronary stenosis to give the "corrected FFR" (cFFR) and percentage of values, which crossed a threshold of 0.8 .

Results: The mean height from coronary ostium to distal left anterior descending (LAD) was $+5.26 \mathrm{~cm}$, distal circumflex $(C x)-3.35 \mathrm{~cm}$, distal right coronary artery-posterior left ventricular artery (RCA-PLV) $-5.74 \mathrm{~cm}$ and distal RCA-posterior descending artery $(P D A)+1.83 \mathrm{~cm}$. For LAD, correction resulted in a mean change in FFR of $+0.042,-0.027$ in the CX, -0.046 in the PLV and +0.015 in the PDA. Using 200 random FFR values between 0.75 and 0.85 , the resulting $c F F R$ crossed the clinical treatment threshold of 0.8 in $43 \%$ of $L A D, 27 \%$ of $C x, 47 \%$ of PLV and $15 \%$ of PDA cases.

Conclusions: There are significant vertical height differences between the distal artery $(P d)$ and its point of normalization (Pa). This is likely to have a modest effect on FFR, and correcting for this results in a proportion of values crossing treatment thresholds. Operators should be mindful of this phenomenon when interpreting FFR values. (Cardiol J 2021; 28, 1: 41-48)
\end{abstract}

Key words: hydrostatic pressure, computed tomography coronary angiography, coronary stenosis

\section{Introduction}

Fractional flow reserve (FFR) is the gold standard for invasive assessment of flow limitation caused by a coronary stenosis and it has been shown to improve clinical outcomes in randomized clinical trials [1-3]. In practice, FFR is calculated as the ratio of the distal trans-stenotic pressure to the proximal coronary or aortic pressure during pharmacological hyperemia. The hydrostatic consequences of the wire position are one of the recognized pitfalls when FFR measurements are performed. Coronary arteries lie in different vertical planes and height variations are part of

Address for correspondence: Dr. Firas Al-Janabi, Essex Cardiothoracic Center, Nethermayne, Basildon, Essex,

United Kingdom, SS16 5NL, tel: +00441268 394155, e-mail: firas.aljanabi@btuh.nhs.uk

Received: 23.07.2018

Accepted: 28.10.2018

This article is available in open access under Creative Common Attribution-Non-Commercial-No Derivatives 4.0 International (CC BY-NC-ND 4.0) license, allowing to download articles and share them with others as long as they credit the authors and the publisher, but without permission to change them in any way or use them commercially. 
normal anatomy. Thus, the pressure wire sensor measuring distal pressure $(\mathrm{Pd})$ is seldom at the same level with the coronary ostium where aortic pressure $(\mathrm{Pa})$ is measured and where the $\mathrm{Pd}$ and $\mathrm{Pa}$ were previously equalized. This effect is present in any pressure based measurement, including the resting indices such as instantaneous wave free ratio (iFR) [4]. Despite strong evidence for its use, FFR remains underutilized [5]. Avoiding confounding factors when using pressure-based indices is crucial in accurate stenosis assessment.

In clinical practice hydrostatic effect produces FFR values higher than 1.00 in a non-diseased vessels, most commonly positioned posteriorly [6]. A recent study documented coronary ostia and distal vessels height differences in an elderly patient cohort with aortic stenosis [7]. Furthermore, the investigators used an in vitro model to calculate the impact of their observed height difference in pressure derived physiological indices. The observed changes were small, meaning that it is unlikely to cause a significant change of FFR value in clinical practice. However, when using a binary cut-off for flow limitation for a given coronary stenosis, even a change of 0.02 can change the classification of FFR from ischemic to non-ischemic (FFR from 0.79 to 0.81 ).

In this study, the aim was to quantify the height differences between distal coronary vessels and corresponding coronary ostia in a supine position in a real-life cohort of patients undergoing investigations for coronary artery disease. Based on these measurements, quantifying the effect of coronary anatomical variations on FFR values around the ischemic cut-off point of 0.80 was sought.

\section{Methods}

A retrospective analysis of 100 patients was conducted who were undergoing computed tomography (CT) coronary angiograms from August 2016 to April 2017 for new onset chest pain suspected to be angina. Vertical coronary height measurements were recorded in all coronary arteries and then used to calculate the potential hydrostatic effect on that specific point in the artery. The effect of the calculated pressure difference and hence effect on FFR was applied to a model of 200 randomly generated FFR values. FFR was compared pre- and post-correction for hydrostatic force.

\section{Inclusion and exclusion criteria}

All patients were elective outpatients under investigation for angina. Patients with previous bypass grafting or valve surgery were excluded. Scans, which did not show the upper rim of the CT table could not be analyzed (as this was the reference point for measurement). Coronary visualizations with poor contrast penetration, or significant artefact were excluded. Finally, left dominant coronary circulations were not included in the present analysis.

\section{CT coronary angiogram}

Computed tomography coronary angiography was performed as per local criteria at the documented institution using a 64-slice CT scanner. A resting heart rate of less than $80 \mathrm{bpm}$ was required. Intravenous metoprolol was administered for heart rate reduction if necessary.

\section{Coronary height analysis}

Using an electronic radiology reporting program (Agfa IMPAX ${ }^{\mathrm{TM}}$ ) and a measuring caliper, distance from the upper rim of the CT table to multiple points in the coronary tree were obtained. Arterial measurement points included:

- left coronary ostium;

- right coronary ostium;

- ostial left anterior descending (LAD);

- distal LAD - at its highest point;

- distal circumflex $(\mathrm{Cx})$ - at its lowest point;

- right coronary artery (RCA) bifurcation;

- distal posterior descending artery (PDA) - at its highest point;

- distal posterior left ventricular artery (PLV) - at its lowest point.

Measurements were in millimeters and taken at the furthest point of contrast penetration visible in the vessel.

\section{FFR impact analysis}

The difference in height between the coronary ostium and the measurement point in the artery is the calculated height difference. This was multiplied by 0.8 (according to the Pascal Law and adjusting for blood density) to give a positive or negative change in pressure - in $\mathrm{mmHg}$. This is the theoretical effect on Pd. The denominator $(\mathrm{Pa})$ is assumed to be 100 in the following calculation model. The resulting value was factored into 200 random computer generated FFR values between 0.75 and 0.85 to give a corrected FFR (cFFR) using Microsoft Excel ${ }^{\mathrm{TM}}$. Corrected FFR was compared with baseline FFR and the percentage of values that crossed the threshold of 0.8 (from positive to negative or vice versa) was calculated. 


\section{Statistical analysis}

Continuous variables are expressed as mean values plus or minus standard deviation. Categorical variables are described as numbers and percentages. Statistical significance of coronary height variations was calculated using the Student t-test.

\section{Results}

\section{Study population}

Patient demographics are summarized in Table 1. All patients had a resting heart rate below $80 \mathrm{bpm}$ before scanning.

\section{Coronary height data}

Figure 1 shows an example of coronary height measurement. The measuring caliper in green calculates height from the upper rim of the CT table to the corresponding point in the coronary artery. In this particular example the caliper is measuring from the ostial left main stem.

Results are displayed below are of all measurement points within the coronary tree (Fig. 2, Table 2). Height measurement is taken from the upper rim of the CT table.

Table 3 summarizes data points from each coronary artery with regard to their respective coronary ostia. The height difference between the coronary specific coronary ostium $(\mathrm{Pa})$ and the vessel containing the height measurement point $(\mathrm{Pd})$, is the value used to calculate effect on FFR and hence, the $\mathrm{cFFR}$.
Table 1. Demographics of 100 study patients.

\begin{tabular}{lc}
\hline Characteristic & $\begin{array}{c}\text { Number } \\
\text { (also \% as } \mathbf{n}=100 \text { ) }\end{array}$ \\
\hline Age & $55.9 \%$ \\
Female & 68 \\
Current smoker & 12 \\
Ex-smoker & 19 \\
Hypertension & 33 \\
Hypercholesterolemia & 25 \\
Family history & 24 \\
Ejection fraction & $54.8 \%$ \\
\hline
\end{tabular}

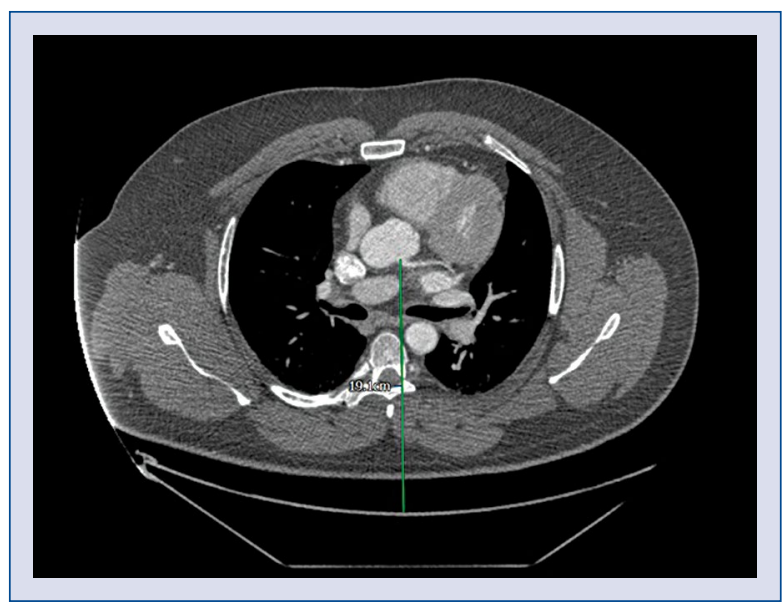

Figure 1. Vessel height measurement illustration on coronary computed tomography. The image demonstrates the measurement caliper from the left main stem ostium, to the upper rim of the computed tomography table.

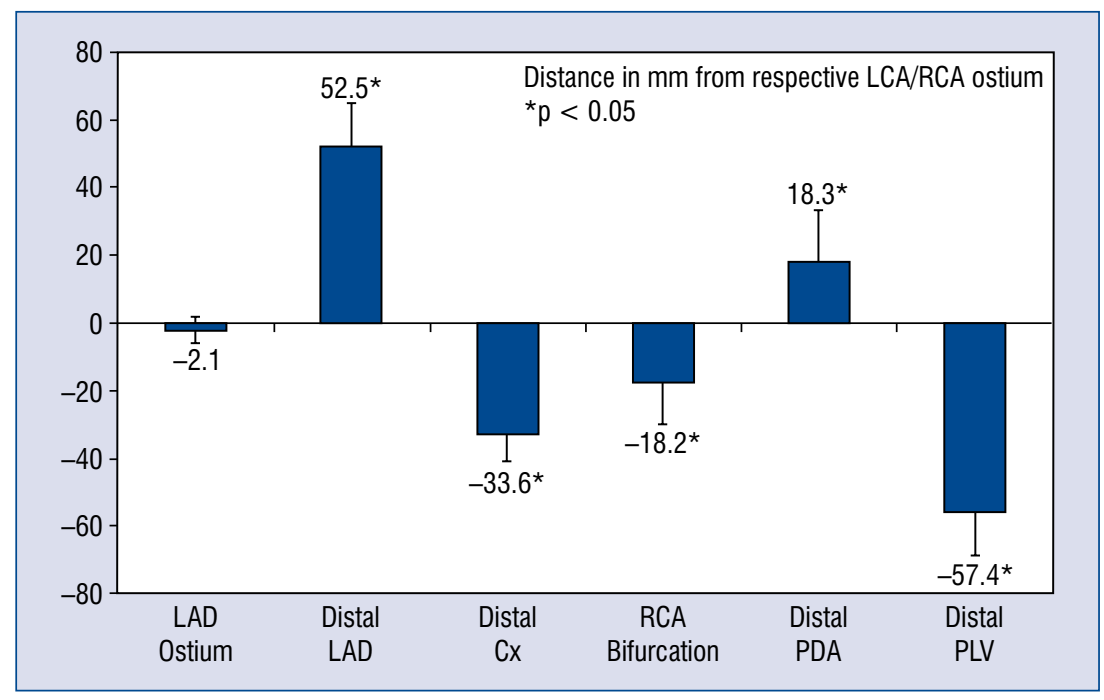

Figure 2. Coronary height variation from their respective ostium. Height variation of the distal vessel from its respective ostium; *These measurements were statistically significant. LAD - left anterior descending; Cx - circumflex; PDA — posterior descending artery; PLV — posterior left ventricular artery; RCA — right coronary artery; LCA — left coronary artery. 
Table 2. Computed tomography (CT) height measurements. The vertical height measurements are shown from the upper rim of the CT table. P values are calculated for each point to the respective vessel ostium.

\begin{tabular}{lcc}
\hline Measurement point & $\begin{array}{c}\text { Mean height from upper } \\
\text { rim of CT table [mm] }\end{array}$ & $\begin{array}{c}\text { P value compared } \\
\text { to vessel ostium }\end{array}$ \\
\hline Left coronary circulation & $170.0 \pm 19.6$ & NA \\
LCA ostium & $167.9 \pm 19.6$ & 0.06 \\
LAD ostium & $222.5 \pm 28.3$ & $<0.0001$ \\
Distal LAD & $136.4 \pm 20.4$ & $<0.0001$ \\
Distal Cx & & \\
Right coronary circulation & $193.8 \pm 21.2$ & $\mathrm{NA}$ \\
RCA ostium & $175.6 \pm 28.3$ & $<0.0001$ \\
RCA bifurcation & $212.1 \pm 30.7$ & $<0.0001$ \\
Distal PDA & $136.4 \pm 26.1$ & $<0.0001$ \\
Distal PLV & & \\
\hline
\end{tabular}

LAD — left anterior descending; LCA — left coronary artery; Cx — circumflex; PDA — posterior descending artery; PLV — posterior left ventricular artery; RCA — right coronary artery; NA — not available

Table 3. Fractional flow reserve (FFR) effect. The height variations have been converted into pressure effect in $\mathrm{mmHg}$. The impact on FFR with a proximal pressure of 100 is shown in the far-right column.

\begin{tabular}{lccc}
\hline Measurement point & $\begin{array}{c}\text { Height from respective } \\
\text { coronary ostium [mm] }\end{array}$ & $\begin{array}{c}\text { Height effect on } \\
\text { distal pressure [mmHg] }\end{array}$ & $\begin{array}{c}\text { FFR correction } \\
\text { factor }\end{array}$ \\
\hline Height from left coronary ostium & +2.1 & -0.2 & +0.002 \\
LAD ostium & +52.5 & +4.2 & +0.04 \\
Distal LAD & -33.6 & -2.7 & -0.03 \\
Distal CX & & & -0.02 \\
Height from right coronary ostium & -18.2 & -1.5 & +0.02 \\
RCA bifurcation & +18.3 & +1.5 & -0.05 \\
Distal PDA & -57.4 & -4.6 & \\
Distal PLV & & -1.6 & \\
\hline
\end{tabular}

LAD - left anterior descending; Cx — circumflex; PDA — posterior descending artery; PLV — posterior left ventricular artery; RCA — right coronary artery

\section{Hydrostatic effect and cFFR}

The corresponding hydrostatic effect of distal LAD, distal Cx, distal PDA and distal PLV were factored into the FFR equation to give the cFFR (Table 3). For anterior vessels, the FFR increased, for posterior vessels, it fell. Out of the 200 randomly generated FFR values, $45.5 \%$ were below 0.8 and $55.5 \%$ above 0.8 . After correction and calculation of cFFR, these percentages changed substantially. Those that crossed from positive to negative, or vice versa were calculated. Table 4 summarizes the results.

\section{Clinical case example}

An in vivo example demonstrating the effect of wire position is presented of a 73-year-old male with a lesion in the mid RCA (Fig. 3). The patient presented with typical stable angina. There was a background history of inflammatory bowel disease, but no typical cardiac risk factors were presented. Ejection fraction was normal. A combined pressure and velocity wire (Combowire, Volcano Corporation $^{\mathrm{TM}}$, San Diego, California, USA) was passed through a $6 \mathrm{~F}$ guiding catheter. The wire was passed beyond the lesion and FFR was measured firstly in the PDA (as distal as a clear velocity tracing allowed), followed by the PLV (distally as per PDA) and lastly three vessel diameters were placed beyond the stenosis in the main mid RCA. $400 \mu \mathrm{g}$ of intra-arterial nitrates were administered before FFR measurement. Intravenous adenosine at $140 \mu \mathrm{g} / \mathrm{kg}$ was used to induce a steady state 
Table 4. Effect on fractional flow reserve (FFR) measurements between 0.75 and 0.8 . The effect on 200 randomly generated FFR measurements is shown for each vessel point. Percent values crossing a threshold of 0.8 is shown in the far-right column.

\begin{tabular}{lccccc}
\hline $\begin{array}{l}\text { Vessel point (+change } \\
\text { in distal pressure) }\end{array}$ & $\begin{array}{c}\text { \% FFR } \\
\text { below 0.8 }\end{array}$ & $\begin{array}{c}\text { \% FFR } \\
\text { above 0.8 }\end{array}$ & $\begin{array}{c}\text { \% cFFR } \\
\text { below 0.8 }\end{array}$ & $\begin{array}{c}\text { \% cFFR } \\
\text { above 0.8 }\end{array}$ & $\begin{array}{c}\text { \% Crossing } \\
\mathbf{0 . 8}\end{array}$ \\
\hline Distal LAD (-0.04) & & 6 & 94 & 42.5 \\
Distal CX (+0.03) & 45.5 & 54.5 & 72 & 28 & 26.5 \\
Distal PLV (+0.05) & & 92 & 8 & 46.5 \\
Distal PDA (-0.02) & & & 30.5 & 69.5 & 15 \\
\hline
\end{tabular}

cFFR - corrected FFR; LAD — left anterior descending; Cx — circumflex; PDA — posterior descending artery; PLV — posterior left ventricular artery

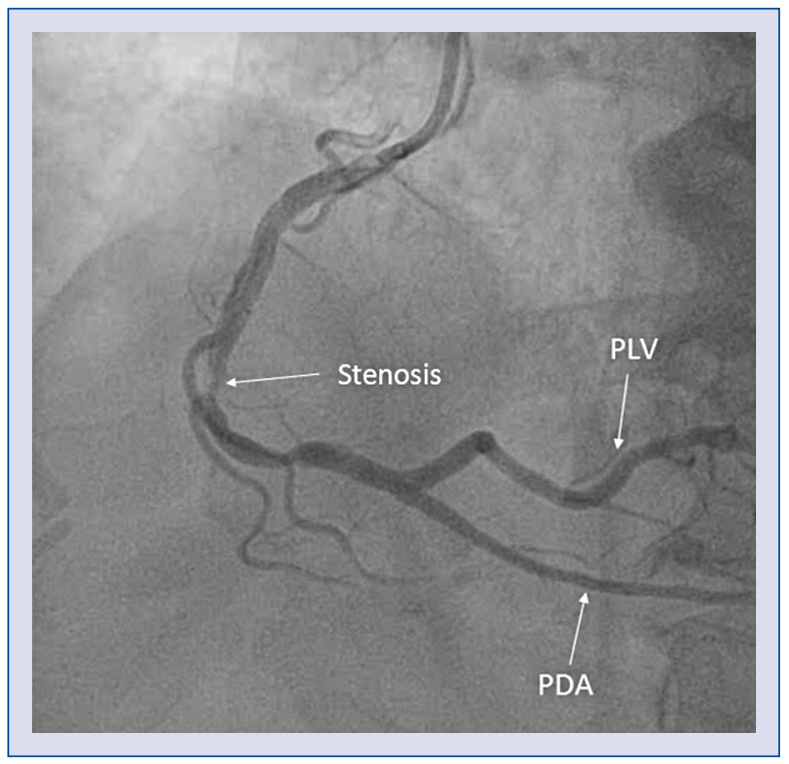

Figure 3. Mid right coronary artery stenosis. The stenosis is shown in the mid right coronary artery, with arrows indicating the posterior left ventricular artery (PLV) and posterior descending artery (PDA).

Table 5. Clinical case data. Fractional flow reserve (FFR) measurement varied by 0.05 between PLV and PDA. Velocity measurements did not vary significantly. This is due to the vertical height differences in both vessels and in turn the hydrostatic effect.

\begin{tabular}{lcc}
\hline Measurement point & FFR & Flow $[\mathrm{cm} / \mathrm{s}]$ \\
\hline PDA & 0.75 & 17.1 \\
PLV & 0.8 & 19.1 \\
Three vessel diameters & 0.79 & 18.6 \\
beyond stenosis & & \\
(mid RCA) & & \\
\hline
\end{tabular}

PDA - posterior descending artery; PLV — posterior left ventricular artery; RCA — right coronary artery of hyperemia. There was no drift with any of the acquired measurements. Invasive measurements are presented in Table 5 .

For the same lesion, placement of the wire in the PDA or PLV altered FFR by 0.05 . Placing the wire three vessel diameters beyond the stenosis, gave an FFR of 0.79 . The small flow variations measured on each occasion were not significantly different, and within normal variations as expected during Doppler measurements [8].

\section{Discussion}

In summary, the present findings show that coronary anatomy results in statistically significant height variations between proximal $(\mathrm{Pa})$ and distal vessel (Pd). There is a potential change in FFR of 0.02-0.05, causing a number of 'grey-zone' FFR results to cross a binary cut-off point.

In the current cohort, the most superior points in a supine patient were the distal LAD, followed by distal PDA. The most inferior points were the distal Cx and distal PLV. All measurements were statistically significant when compared to the respective ostium, apart from the ostial LAD. Even though mean height of PLV and Cx were identical with reference to the $\mathrm{CT}$ table, when compared to their respective ostium $(\mathrm{Pa})$, the PLV had a larger height difference, owing to the more superior position of the RCA ostium. In turn, the hydrostatic pressure effect was more pronounced in the PLV. More proximal points in a vessel, e.g. ostial LAD or RCA bifurcation had a smaller height variation when compared to their respective coronary artery ostium. In general, there was a gradual change in height from proximal to distal vessel. Note however, that the most distal point in the vessel does not always have the greatest height variation. An example of this is in a 'wrap around' LAD, where 
the vessel height falls after reaching the apex. This occurred in over half of patients in one study [9].

Computed tomography coronary angiography can accurately map the course of coronary vessels and their vertical heights. Subsequently, the height of the distal vessel (i.e. the position of the pressure wire, or $\mathrm{Pd}$ ) may be higher, or lower than its origin $(\mathrm{Pa})$, depending on the course it takes. This may explain observed changes in groups of patients with 'moderate' coronary stenoses in which posterior vessels (those vertically lower when supine - circumflex, posterior left ventricular) have higher mean FFR values than anterior vessels (those that are vertically higher - left anterior descending, posterior descending) [10]. Resting $\mathrm{Pd} / \mathrm{Pa}$ can also often be seen above 1.0. Studies have identified this phenomenon [6] and it is caused by the distal pressure sensor sitting vertically lower than the aortic pressure sensor (and original point of normalization). For a resting index to be above one, disease in the vessel is usually mild. While often attributed to drift, physical principles can predict this concept. It is useful to note this phenomenon rather than to assume the physiology wire is at fault.

A recent study assessing coronary artery height variations using CT coronary angiograms has been conducted recently in a group composed predominantly of transcutaneous aortic valve implantation patients [5]. Hydrostatic pressure effects were then confirmed using an in vitro model. The anatomy of these patients with severe aortic stenosis may slightly alter the anatomy of the coronary arteries themselves due to changes in the aortic root. The present assessment of coronary height variations in a more heterogeneous group of patients presenting with stable cardiac chest pain was thought to be a useful addition to current knowledge. In general, patients studied herein were younger females in keeping with the low to intermediate risk group initially assessed with CT coronary angiography at the time. There were some differences in height measurements from CT scans between this study and Härle et al. [10]. Measurements from ostial left coronary artery to $\mathrm{LAD}$ and $\mathrm{Cx}$ were similar (5.3 vs. $4.9 \mathrm{~cm}$ and 3.4 vs. $3.9 \mathrm{~cm}$, respectively). There were however more pronounced differences in the measurement of PLV and PDA from the right coronary ostium (5.7 vs. 2.6 and 1.8 vs. 3.8). There are potential explanations. Observer variation between two studies may account for some of the change. Contrast penetration into the distal vessel can significantly alter the measurement point within the artery, thus leading to errors in measurement in both studies. Finally, the patient cohort varies between the studies. One anticipates that coronary height measurements may vary between a predominantly older population with aortic stenosis, and a younger cohort without.

Pressure based invasive physiology such as FFR, has been well validated for many years. However, pressure-based measurements are subject to potential effects of hydrostatic pressure. If hydrostatic forces alter distal pressure recordings FFR will in turn change. The change may be small (0.02-0.05) but useful to acknowledge for FFR values circling the cut-off point $(0.75-0.85)$ [11]. In theory, the addition of adenosine should not alter the physical hydrostatic pressure effect in a coronary vessel in vivo, as height, fluid density and gravitational effect have not changed. An important consideration is the hypotensive effect and hence reduction in Pa during adenosine infusion. Aortic pressure may fall below $100 \mathrm{mmHg}$ during hyperemia, meaning alterations in $\mathrm{Pd}$ have a larger effect on overall $\mathrm{Pd} / \mathrm{Pa}$. Hydrostatic effect is constant across resting and hyperemic states. A change in $\mathrm{Pd}$ of $5 \mathrm{mmHg}$ is therefore of greater relative importance in resting indices (where a transtenotic gradient of $10 \mathrm{mmHg}$ is considered abnormal) compared to hyperemic indices (where $20 \mathrm{mmHg}$ is considered abnormal).

Whilst the effect of hydrostatic pressure upon FFR is described, it was believed herein, that this novel data demonstrates that depending on the coronary artery in question and its anatomical course the physiological significance of coronary stenosis can be both over or under-estimated. Treatment of intermediate coronary stenoses therefore must not be a binary decision, and the operator must exert clinical judgment when faced with a grey zone physiological values.

The exact position of the pressure sensor of the physiology wire is often not considered. Hydrostatic effect becomes more pronounced as the pressure sensor is positioned more distally. Avoiding an unnecessarily distal wire position will minimize the hydrostatic effect on obtained measurements by reducing the guide to pressure sensor distance.

By changing patient position during angiography, (i.e. turning onto one side), and leaving the wire in exactly the same position in the artery, FFR values have been shown to change [12]. Correcting for the presumed hydrostatic effect due to this position change (by using measured height difference between guide and wire), abolished the 
difference between the two FFR recordings, seeming to explain the difference.

Another important observation is the pressure change along the longitudinal length of a coronary artery, which has been attributed to diffuse atherosclerosis [13]. The additive effect of hydrostatic pressure however cannot be excluded, as vertical height also gradually changes along the length of an artery. This along with other confounding factors, such chronic kidney disease [14] may also impact stenosis assessment. Finally, hydrostatic pressure effects may also contribute to measurements that use mean distal pressure, such as the index of microvascular resistance measured using thermodilution.

In the present clinical case example, wire placement altered FFR by 0.05 (PDA vs. PLV placement). Flow within the artery does not change in this case study as coronary autoregulation maintains flow over a wide pressure range when these mechanisms are intact [15]. Using the present coronary CT data, the mean height difference between PLV and PDA was $7.57 \mathrm{~cm}$, equating into a potential distal pressure difference $(\mathrm{Pd})$ of 6.06 $\mathrm{mmHg}$. Therefore, a change in FFR of up to 0.06 is possible on average. This is the mean change, and patient factors such as height, play a role in individual FFR measurements [7]. Although clinical decision-making takes into account multiple factors and is not a binary process revolving around a cut-off point, one should recognize the potential effects of wire position and hydrostatic pressure.

\section{Limitations of the study}

The study group consisted of low to intermediate risk patients, hence, the majority were younger females. This is not in keeping with typical demographics of patients who require invasive treatment for coronary artery disease.

The visualization of the coronary artery in question was limited by contrast penetration into the distal vessel. Some vessels were not completely opacified, meaning a potential underestimation of height measurements were present. This seemed especially prominent in the PDA where contrast did not penetrate to the distal vessel in $15 \%$ of cases. Measurements for these patients were excluded.

Height was measured at distal sections in the coronary artery, as this was the point of maximal height variation. In clinical practice the wire is often not positioned as far distal as these measurements were taken, meaning there was a potential overestimation of the hydrostatic effect.
With regard to the 200 random FFR results generated, it can be seen that $54.5 \%$ of FFR values generated were over 0.8 . This was apparently a chance occurrence, but the lack of a more linear 50/50 split of values will affect subsequent analyses.

The hydrostatic effect on FFR in this study takes into account Pa pressure of $100 \mathrm{mmHg}$. Further data on alterations in $\mathrm{Pa}$ and the subsequent impact on FFR may have been a useful addition.

The calculated hydrostatic effect is theoreti$\mathrm{cal}$, and needs further investigation in vivo. Recent trials have upheld anticipated changes in pressure based measurements due to hydrostatic forces [12].

\section{Conclusions}

The anatomical path of coronary arteries resulted in a significant vertical height difference between the distal artery (Pd) and its point of normalization $(\mathrm{Pa})$. According to the present hydrostatic pressure model, this is likely to have a modest effect on FFR calculation, which in turn, could result in values crossing the treatment threshold. Operators should be mindful of this phenomenon when interpreting FFR values, particularly in the LAD and RCA-PLV.

\section{Acknowledgements}

We thank the authors for their contribution in production of this manuscript.

\section{Conflict of interest: None declared}

\section{References}

1. Zimmermann FM, Ferrara A, Johnson NP, et al. Deferral vs. performance of percutaneous coronary intervention of functionally non-significant coronary stenosis: 15 -year follow-up of the DEFER trial. Eur Heart J. 2015; 36(45): 3182-3188, doi: 10.1093/ eurheartj/ehv452, indexed in Pubmed: 26400825.

2. Pijls NHJ, Fearon WF, Tonino PAL, et al. Fractional flow reserve versus angiography for guiding percutaneous coronary intervention in patients with multivessel coronary artery disease: 2-year follow-up of the FAME (Fractional Flow Reserve Versus Angiography for Multivessel Evaluation) study. J Am Coll Cardiol. 2010; 56(3): 177-184, doi: 10.1016/j.jacc.2010.04.012, indexed in Pubmed: 20537493.

3. Xaplanteris P, Fournier S, Pijls NHJ, et al. Five-Year outcomes with PCI guided by fractional flow reserve. N Engl J Med. 2018; 379(3): 250-259, doi: 10.1056/NEJMoa1803538, indexed in Pubmed: 29785878.

4. Davies JE, Sen S, Dehbi H-M, et al. Use of the Instantaneous Wave-free Ratio or Fractional Flow Reserve in PCI. N Engl J Med. 2017; 376(19): 1824-1834. 
5. Tebaldi M, Biscaglia S, Fineschi M, et al. Evolving Routine Standards in Invasive Hemodynamic Assessment of Coronary Stenosis: The Nationwide Italian SICI-GISE Cross-Sectional ERIS Study. JACC Cardiovasc Interv. 2018; 11(15): 1482-1491, doi: 10.1016/j.jcin.2018.04.037, indexed in Pubmed: 29803695.

6. Nijjer SS, de Waard GA, Sen S, et al. Coronary pressure and flow relationships in humans: phasic analysis of normal and pathological vessels and the implications for stenosis assessment: a report from the Iberian-Dutch-English (IDEAL) collaborators. Eur Heart J. 2016; 37(26): 2069-2080, doi: 10.1093/eurheartj/ ehv626, indexed in Pubmed: 26612582.

7. Härle T, Luz M, Meyer S, et al. Effect of coronary anatomy and hydrostatic pressure on intracoronary indices of stenosis severity. JACC Cardiovasc Interv. 2017; 10(8): 764-773, doi: 10.1016/j. jcin.2016.12.024, indexed in Pubmed: 28365266.

8. Davies JE, Whinnett ZI, Francis DP, et al. Evidence of a dominant backward-propagating "suction" wave responsible for diastolic coronary filling in humans, attenuated in left ventricular hypertrophy. Circulation. 2006; 113(14): 1768-1778, doi: 10.1161/CIRCULATIONAHA.105.603050, indexed in Pubmed: 16585389.

9. Kobayashi N, Maehara A, Brener SJ, et al. Usefulness of the Left Anterior Descending Coronary Artery Wrapping Around the Left Ventricular Apex to Predict Adverse Clinical Outcomes in Patients With Anterior Wall ST-Segment Elevation Myocardial Infarction (from the Harmonizing Outcomes With Revascularization and Stents in Acute Myocardial Infarction Trial).
Am J Cardiol. 2015; 116(11): 1658-1665, doi: 10.1016/j.amjcard.2015.09.004, indexed in Pubmed: 26433272.

10. Härle T, Meyer S, Bojara W, et al. Intracoronary pressure measurement differences between anterior and posterior coronary territories. Herz. 2017; 42(4): 395-402, doi: 10.1007/s00059016-4471-z, indexed in Pubmed: 27582367.

11. Petraco R, Escaned J, Sen S, et al. Classification performance of instantaneous wave-free ratio (iFR) and fractional flow reserve in a clinical population of intermediate coronary stenoses: results of the ADVISE registry. EuroIntervention. 2013; 9(1): 91-101, doi: 10.4244/EIJV9I1A14, indexed in Pubmed: 22917666.

12. Härle T, Luz M, Meyer S, et al. Influence of hydrostatic pressure on intracoronary indices of stenosis severity in vivo. Clin Res Cardiol. 2018; 107(3): 222-232, doi: 10.1007/s00392-017-1174-2, indexed in Pubmed: 29098379.

13. De Bruyne B, Hersbach F, Pijls NH, et al. Abnormal epicardial coronary resistance in patients with diffuse atherosclerosis but "Normal" coronary angiography. Circulation. 2001; 104(20): 2401-2406, indexed in Pubmed: 11705815.

14. Tebaldi M, Biscaglia S, Fineschi M, et al. Fractional flow reserve evaluation and chronic kidney disease: analysis from a multicenter italian registry (the FREAK study). Catheter Cardiovasc Interv. 2016; 88(4): 555-562, doi: 10.1002/ccd.26364, indexed in Pubmed: 26717890.

15. Ramanathan T, Skinner H. Coronary blood flow. Contin Educ Anaesth Crit Care Pain. 2005; 5(2): 61-64, doi: 10.1093/bjaceaccp/mki012. 